\title{
Etude de quelques souches de levures isolées du fromage
}

\author{
par \\ G. MOULIN, P. GALZY et F. PERRON* \\ Laboratoire de Recherches de la Chaire de Génétique \\ Ecole Nationale Supérieure Agronomique \\ Centre de Recherches Agronomiques de Montpellier \\ Institut National de la Recherche Agronomique \\ (34) Montpellier (France)
}

\section{INTRODUCTION}

Certaines levures (Kluyveromyces fragilis) sont utilisées pour traiter le lactosérum et pour préparer une levure aliment de bonne qualité (brevet 1128063 des fromageries Bel). Afin d'améliorer la transformation du lactosérum un travail a été entrepris pour sélectionner des souches nouvelles. Une première étude a été effectuée en utilisant des souches de Kluyveromyces issues de diverses collections (Ladet et al., 1972). Nous résumons ici les résultats obtenus en étudiant des souches isolées de diverses fabrications fromagères.

\section{MATERIEL ET METHODES}

\section{A) Matériel biologique}

Neuf souches de levure ont été étudiées. Les souches $\mathrm{D}_{1}$ et $\mathrm{D}_{2}$ proviennent de fromage blanc frais. Les souches $\mathrm{OCH} 1, \mathrm{OCH} 2, \mathrm{O} 2 \mathrm{CH}$, $\mathrm{DCH}$ et $\mathrm{YMCH}$ ont été isolées sur cheddar jeune. Enfin, les souches YCRV et OCRV proviennent d'une fabrication expérimentale du Centre de recherches des Fromageries Bel de Vendôme.

\section{B) Techniques de culture}

Les souches sont conservées sur yeast extract Difco 0,5 p. 100, glucose 0,5 p. 100 , gélosé à 2 p. 100. Les tests physiologiques sont effectués sur yeast nitrogen base Difco avec du lactose comme seule

\footnotetext{
* Fromagerie Picon (74) Saint-Félix.
} 
source de carbone. Les cultures sont conduites en erlenmeyers remplis au dixième du volume et agitées (amplitude $7 \mathrm{~cm} ; 80$ oscillations par $\mathrm{mn}$ ).

Les tests taxonomiques ont été effectués selon J. Lodder (1970).

\section{C) Techniques analytiques}

Les protéines sont dosées par la réaction du biuret selon Strickland (1951).

La matière sèche est mesurée par pesée après dessiccation à $108^{\circ} \mathrm{C}$ jusqu'à poids constant.

Le nombre de cellules est estimé par comptage hématimétrique.

Les lipides totaux, l'insaponifiable et le saponifiable sont extraits et dosés par les méthodes déjà décrites (A. Chassang et al., 1972).

\section{RESULTATS EXPERIMENTAUX}

\section{A) Etude taxonomique}

L'étude taxonomique a montré que les 9 souches étudiées pouvaient être rassemblées en trois groupes. En effet, les souches $D_{1}$ et $\mathrm{D}_{2}$ sont pratiquement identiques. Il en est de même des souches OCH1, DCH, 02CH, OCH2 d'une part et des souches OCRV, YCRV, YMCH d'autre part. Les résultats sont groupés dans les tableaux 1,2 et 3.

Les souches $\mathrm{D}_{1}$ et $\mathrm{D}_{2}$ présentent une multiplication végétative par bourgeonnement multilatéral et sont sporogènes. Elles appartiennent à la tribu des Saccharomyceteae. Elles diffèrent des Kluyveromyces par la résistance de leur asque et par leur aptitude à se multiplier en l'absence de vitamines. Elles diffèrent des Pichia par la vigueur du métabolisme fermentaire, l'absence de pseudomycélium et l'absence de voile et d'anneau.

En définitive ces souches semblent appartenir au genre Saccharomyces. 1970).

Elles sont différentes de toutes les espèces décrites (J. Lodder,

Elles assimilent le lactose contrairement aux autres Saccharomyces.

Les souches $\mathrm{O} 2 \mathrm{CH}, \mathrm{OCH} 1, \mathrm{OCH} 2$ et $\mathrm{DCH}$ présentent un bourgeonnement multilatéral et sont anascosporogènes. Elles appartiennent au genre Torulopsis. Elles sont voisines de Torulopsis candida bien que leurs cellules soient plus petites.

Enfin, les souches OCRV, YCRV et YMCH sont également anascosporogènes et présentent un bourgeonnement multilatéral. Elles sont très 
TABLEAU 1

\begin{tabular}{|c|c|c|c|}
\hline Souches & $D_{1}-D_{2}$ & $\begin{array}{c}\mathrm{OCH} 1-\mathrm{OCH} 2 \\
\mathrm{O} 2 \mathrm{CH}-\mathrm{DCH}\end{array}$ & $\begin{array}{c}\text { OCRV - YCRV } \\
\text { YMCH }\end{array}$ \\
\hline $\begin{array}{l}\begin{array}{l}\text { Morphologie cellulaire } \\
\text { Colonies }\end{array} \\
\text { Milieu liquide } \\
\text { Ascospores } \\
\text { Assimilation des nitrates } \\
\text { Assimilation des nitrites } \\
\text { Croissance sans vitamines } \\
\text { Croissance en présence de } \\
\text { ClNa } 10 \mathrm{p} .100 \\
\text { Esters } \\
\text { Actidione résistance } \\
\text { Pseudomycélium } \\
\text { Test à l'iode } \\
\text { Croissance sur yeast extract } \\
\text { glucose } 50 \mathrm{p} \text {. } 100 \mathrm{P} / \mathrm{P} \\
\text { Croissance } 37^{\circ} \mathrm{C}\end{array}$ & $\begin{array}{c}(1,5-4) \times(2-5) \mu \\
\text { lisses - brillantes } \\
\text { grisâtres } \\
- \text { dépôt } \\
\text { - pas de voile } \\
\text { - pas d'anneau } \\
2 \text { à } 4 \text { spores lisses } \\
\text { pas asque } \\
- \\
- \\
+ \\
+ \\
+ \\
+ \\
+ \\
+ \\
+\end{array}$ & $\begin{array}{l}(1-2) \times(2-4) \mu \\
\text { lisses - brillantes } \\
\text { blanches } \\
- \text { dépôt } \\
- \text { voile mince } \\
- \text { anneau } \\
- \\
= \\
= \\
+ \\
+ \\
= \\
= \\
- \\
+ \\
+\end{array}$ & $\begin{array}{c}(1-3) \times(2-6) \mu \\
\text { lisses - brillantes } \\
\text { blanches } \\
- \text { dépôt } \\
\text { - pas de voile } \\
- \text { anneau } \\
- \\
= \\
+ \\
+ \\
+ \\
= \\
= \\
- \\
+\end{array}$ \\
\hline
\end{tabular}


TABLEAU 2. - Tests d'assimilation et de fermentation

\begin{tabular}{|c|c|c|c|c|}
\hline Souches & $\mathrm{D}_{1}-\mathrm{D}_{2}$ & $\begin{array}{l}\mathrm{OCH} 1-\mathrm{OCH} 2 \\
\mathrm{DCH}-\mathrm{O} 2 \mathrm{CH}\end{array}$ & \multicolumn{2}{|c|}{$\begin{array}{c}\text { OCRV - YCRV } \\
\text { YMCH }\end{array}$} \\
\hline Glucose & $+\mathrm{f}$ & + & + & f. \\
\hline Galactose & $+\mathrm{f}$ & + & + & f. \\
\hline Raffinose & $+\mathrm{f}$ & + & + & \\
\hline Lactose & + & - & + & \\
\hline Saccharose & $+\mathrm{f}$ & + & + & \\
\hline Maltose & + & + & + & \\
\hline Tréhalose & + & + & + & \\
\hline Mélibiose & - & + & + & \\
\hline$\alpha$-Méthyl-D-glucoside & $+\mathrm{f}$ & - & + & \\
\hline f. ' fermentation du substrat & + assi & - & tion & \\
\hline
\end{tabular}


TABLEAU 3. - Tests d'assimilation

\begin{tabular}{|c|c|c|c|}
\hline Souches & $\mathrm{D}_{1}-\mathrm{D}_{2}$ & $\begin{array}{l}\mathrm{O} 2 \mathrm{CH}-\mathrm{OCH} \\
\mathrm{OCH} 2-\mathrm{DCH}\end{array}$ & $\begin{array}{c}\text { OCRV - YCRV } \\
\text { YMCH }\end{array}$ \\
\hline $\begin{array}{l}\text { L. Sorbose } \\
\text { D. Arabinose } \\
\text { L. Rhamnose } \\
\text { Ethanol } \\
\text { Glycérol } \\
\text { Inositol } \\
\text { D. Cellobiose } \\
\text { Inuline } \\
\text { A. Succinique } \\
\text { A. Lactique } \\
\text { Sorbitol } \\
\text { Manitol } \\
\text { D. Ribose } \\
\text { D. Xylose } \\
\text { A. Citrique } \\
\text { Saliciline } \\
\text { Melézitose } \\
\text { Galactitol } \\
\text { Erythritol } \\
\text { Ribitol }\end{array}$ & $\begin{array}{l}+ \\
- \\
+ \\
+ \\
- \\
+ \\
+ \\
+ \\
+ \\
- \\
+ \\
+ \\
+ \\
+ \\
- \\
-\end{array}$ & $\begin{array}{l}+ \\
- \\
+ \\
+ \\
+ \\
+ \\
+ \\
+ \\
+ \\
+ \\
+ \\
+ \\
+ \\
+ \\
+ \\
+ \\
- \\
-\end{array}$ & $\begin{array}{l}+ \\
+ \\
+ \\
+ \\
- \\
+ \\
+ \\
+ \\
+ \\
+ \\
+ \\
+ \\
+ \\
+ \\
+ \\
+ \\
- \\
-\end{array}$ \\
\hline
\end{tabular}



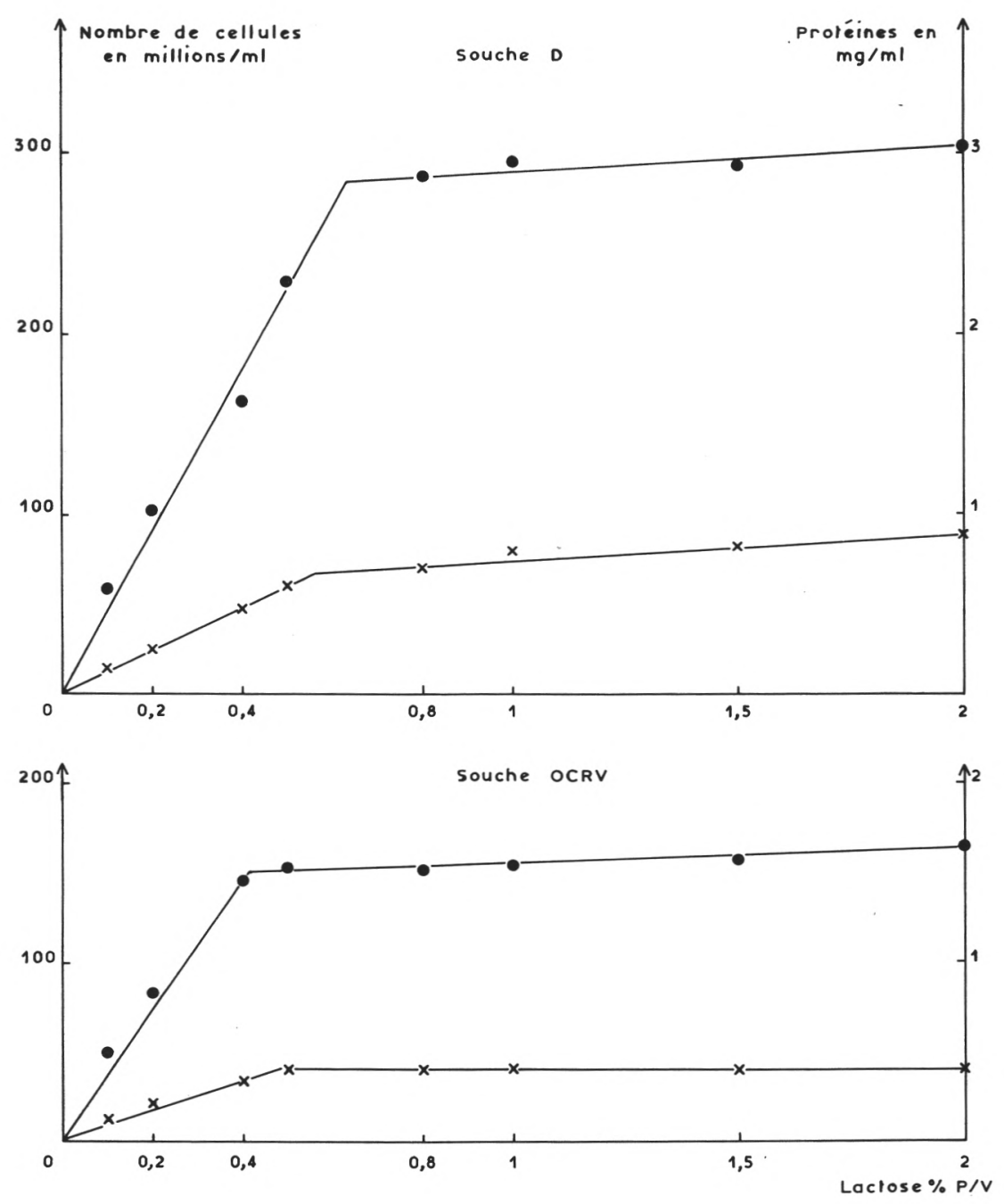

Graphique 1

Etude du rendement de croissance en présence de quantités variables de lactose.

$\times$ Protéines par $\mathrm{ml}$ de culture exprimées en mg.

- Nombre de cellules par ml de culture exprimé en millions. 
voisines des précédentes. Cependant, elles fermentent très légèrement glucose et galactose.

\section{B) Etude du rendement de croissance des souches $D_{1}$ et OCRV}

Le rendement de croissance en présence de lactose a été étudié en faisant varier la concentration de 0,1 p. 100 à 2 p. 100 .

Les résultats sont représentés sur le graphique 1.

Dans le cas de la souche $D_{1}$, le lactose est le seul facteur limitant la croissance sur yeast nitrogen base Difco jusqu'à une concentration de l'ordre de 0,6 p. 100. Le rendement de la croissance a été calculé en se plaçant dans les conditions où le lactose est le facteur limitant. L'utilisation de $1 \mathrm{mg}$ de lactose donne environ :

$0,5.10^{5}$ cellules,

$0,11 \mathrm{mg}$ de protéines,

$0,39 \mathrm{mg}$ de matière sèche.

Dans le cas de la souche OCRV, le lactose est le facteur limitant la croissance jusqu'à une concentration voisine de 0,4 p. 100. L'utilisation de $1 \mathrm{mg}$ de lactose donne environ :
$0,4.10^{\mathrm{s}}$ cellules,
$0,09 \mathrm{mg}$ de protéines,
$0,31 \mathrm{mg}$ de matière sèche.

\section{C) Etude de la composition des cellules}

La composition des cellules des souches $\mathrm{D}_{1}$ et OCRV a été étudiée après culture sur yeast nitrogen base Difco en présence de lactose (tab. 4).

La souche $\mathrm{D}_{1}$ est pauvre en protéines, riche en lipides et notamment en saponifiable. Elle ne présente donc pas d'intérêt industriel.

La souche OCRV est également trop pauvre en protéines et trop riche en lipides pour être utilisée en levurerie.

\section{CONCLUSION}

Notons au passage que ces résultats confirment la grande variété des souches de levures présentes dans le fromage (M. Galzin et al., 1970). Il serait important de déterminer avec précision le rôle des levures dans l'évolution et la maturation des fromages.

Parmi les souches étudiées, $\mathrm{OCH}, \mathrm{OCH} 2, \mathrm{O} 2 \mathrm{CH}, \mathrm{DCH}$ ne présentent pas d'intérêt pour nous puisqu'elles ne fermentent pas le lactose. Les souches OCRV, YMCH, YCRV ont un rendement en protéines sur lactose extrêmement faible. Ces cellules sont plus pauvres en protéines et plus riches en lipides que les Kluyveromyces. 
TABLEAU 4. - Composition des cellules de deux souches après croissance sur lactose

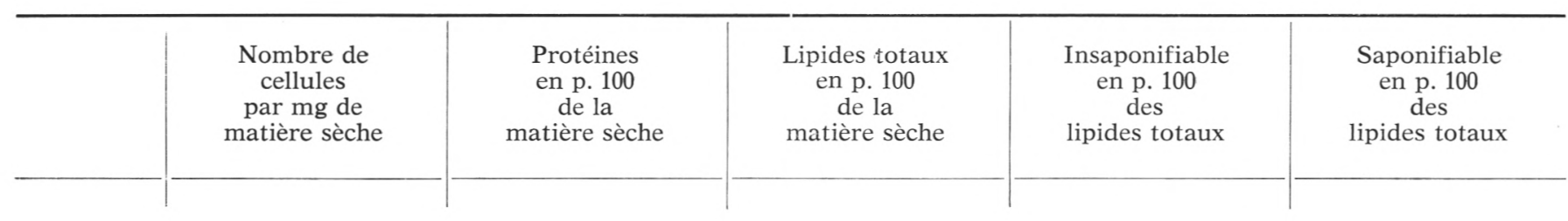

0,2 p. 100 lactose

\begin{tabular}{c|l|l|l|r|}
$\mathrm{D}_{1}$ & 1,2 & 32 & 11 & 15 \\
OCRV & 0,8 & 28 & 9 & 15 \\
48
\end{tabular}

0,8 p. 100 lactose

\begin{tabular}{c|l|l|r|r|r}
$\mathrm{D}_{1}$ & 1,8 & 33 & 12 & 18 \\
OCRV & 0,9 & 30 & 8 & 12 \\
\hline
\end{tabular}


L'ensemble de ces souches n'a pas d'intérêt en levurerie.

Les souches $\mathrm{D}_{1}$ et $\mathrm{D}_{2}$ présentent des caractéristiques meilleures que les précédentes. Cependant, le rendement en protéines obtenu dans les cultures sur lactose est trop faible pour que ces souches soient utilisables en levurerie. Elles présentent des caractéristiques générales inférieures à celles de $K$. fragilis.

\section{S u m m a r y}

Ten strains of yeasts were isolated during the making of some cheeses. They were classed in the genus Torulopsis and Saccharomyces. The growth on lactose and composition of cells were studied.

Reçu pour publication en décembre 1972.

\section{Bibliographie}

Chassang (Aline), Vezinhet (Françoise), Roger (M.) et Galzy (P.) (1971). - Variation de la teneur en lipides des cellules de levures au cours de la sporulation. Folia Microbiologia, Prague (sous presse).

Galzin (M.), Galzy (P.) et BRET (G.). - Etude de la flore de levure dans le fromage de Roquefort. Le Lait, janvier-février 1970, 1-37.

Ladet (J.), Moulin (G.), Galzy (P.), Joux (J. L.) et BiJu-Duval (F.). - Comparaison des rendements de croissance sur lactose de quelques Kluyveromyces Van Der Walt. Le Lait, novembre-décembre 1972, 613-621.

LodDER (J.) (1970). - The yeast a toxonomic study. North Holland Publishing compagny, Amsterdam.

STRICKLAND (1951). - The determination of small quantity of bacteria by means of the biuret reaction. J. Gen Microbiol., 5, 698 . 